\title{
Are increased left ventricular strains compensatory effects in lipedema? Detailed analysis from the three-dimensional speckle-tracking echocardiographic MAGYAR-Path Study
}

\author{
Attila Nemes MD, PhD, DSc, FESC ${ }^{1}$ @ | Árpád Kormányos $\mathrm{MD}^{1}$ \\ Péter Domsik MD, $\mathrm{PhD}^{1} \quad$ | Anita Kalapos MD, $\mathrm{PhD}^{1}$ ｜ Nándor Gyenes $\mathrm{MD}^{1}$ ～ \\ Lajos Kemény MD, PhD, DSc ${ }^{2}$ | Győző Szolnoky MD, PhD
}

\author{
${ }^{1}$ 2nd Department of Medicine and Cardiology
Centre, Faculty of Medicine, Albert Szent-
Györgyi Clinical Center, University of Szeged,
Szeged, Hungary
${ }^{2}$ Department of Dermatology and Allergology,
Faculty of Medicine, Albert Szent-Györgyi
Clinical Center, University of Szeged, Szeged,
Hungary \\ ${ }^{1}$ 2nd Department of Medicine and Cardiology
Centre, Faculty of Medicine, Albert Szent-
Györgyi Clinical Center, University of Szeged,
Szeged, Hungary
${ }^{2}$ Department of Dermatology and Allergology,
Faculty of Medicine, Albert Szent-Györgyi
Clinical Center, University of Szeged, Szeged,
Hungary \\ ${ }^{1}$ 2nd Department of Medicine and Cardiology
Centre, Faculty of Medicine, Albert Szent-
Györgyi Clinical Center, University of Szeged,
Szeged, Hungary
${ }^{2}$ Department of Dermatology and Allergology,
Faculty of Medicine, Albert Szent-Györgyi
Clinical Center, University of Szeged, Szeged,
Hungary \\ ${ }^{1}$ 2nd Department of Medicine and Cardiology
Centre, Faculty of Medicine, Albert Szent-
Györgyi Clinical Center, University of Szeged,
Szeged, Hungary
${ }^{2}$ Department of Dermatology and Allergology,
Faculty of Medicine, Albert Szent-Györgyi
Clinical Center, University of Szeged, Szeged,
Hungary \\ ${ }^{1}$ 2nd Department of Medicine and Cardiology
Centre, Faculty of Medicine, Albert Szent-
Györgyi Clinical Center, University of Szeged,
Szeged, Hungary
${ }^{2}$ Department of Dermatology and Allergology,
Faculty of Medicine, Albert Szent-Györgyi
Clinical Center, University of Szeged, Szeged,
Hungary \\ ${ }^{1}$ 2nd Department of Medicine and Cardiology
Centre, Faculty of Medicine, Albert Szent-
Györgyi Clinical Center, University of Szeged,
Szeged, Hungary
${ }^{2}$ Department of Dermatology and Allergology,
Faculty of Medicine, Albert Szent-Györgyi
Clinical Center, University of Szeged, Szeged,
Hungary \\ ${ }^{1}$ 2nd Department of Medicine and Cardiology
Centre, Faculty of Medicine, Albert Szent-
Györgyi Clinical Center, University of Szeged,
Szeged, Hungary
${ }^{2}$ Department of Dermatology and Allergology,
Faculty of Medicine, Albert Szent-Györgyi
Clinical Center, University of Szeged, Szeged,
Hungary \\ ${ }^{1}$ 2nd Department of Medicine and Cardiology
Centre, Faculty of Medicine, Albert Szent-
Györgyi Clinical Center, University of Szeged,
Szeged, Hungary
${ }^{2}$ Department of Dermatology and Allergology,
Faculty of Medicine, Albert Szent-Györgyi
Clinical Center, University of Szeged, Szeged,
Hungary

\section{Correspondence} \\ Attila Nemes, 2nd Department of Medicine \\ and Cardiology Center, Medical Faculty, Albert \\ Szent-Györgyi Clinical Center, University of \\ Szeged, Semmelweis Street 8, P.O. Box \\ 427, H-6725 Szeged, Hungary. \\ Email: nemes.attila@med.u-szeged.hu
}

Funding information

International Phlebology Union (UIP)Bauerfeind, Grant/Award Number: Award 2015-2017

\begin{abstract}
Purpose: Lipedema is a chronic, common but underdiagnosed disease masquerading obesity, with female predominance, characterized by disproportional abnormal adipose tissue distribution of the lower and also upper extremities. The present study was designed to determine whether lipedema is associated with three-dimensional (3D) speckle-tracking echocardiography (3DSTE)-derived left ventricular (LV) deformation abnormalities, and to assess the effects of 1-hour use of medical compression stockings (MCS).

Methods: The present study comprised 19 female patients with lipedema (mean age: $42.2 \pm 12.4$ years), compared to 28 age-matched healthy female controls (mean age: $42.0 \pm 9.8$ years).

Results: Lipedema patients showed larger left atrial and LV dimensions and greater LV ejection fraction than controls, without significant difference in other echocardiography variables. Lipedema patients had greater 3DSTE-derived global and mean segmental LV circumferential and area strains than controls. Following 1-hour use of wearing MCS, neither global and nor mean segmental LV strains showed significant impairment or improvement.

Conclusions: Increased LV strains could be compensatory effects maintaining LV pumping function in lipedema. Short-term wearing of MCS has no global effect on LV strains.
\end{abstract}

\section{KEYWORDS}

echocardiography, left ventricle, lipedema, speckle-tracking, three-dimensional

\section{1 | INTRODUCTION}

Lipedema is a chronic, common but underdiagnosed and progressive disease masquerading obesity, with female predominance, characterized by disproportional abnormal adipose tissue distribution between the lower and also upper extremities. ${ }^{1,2}$ Although treatment strategies of this disease are debated, the use of medical compression stockings
(MCS) is a part of regular treatment in lipedema. ${ }^{3}$ The development of lipedema may be partly explained by a polygenic background combined with hormonal, microvascular, and lymphatic disorders. ${ }^{4}$ Although patients with lipedema do not display severe cardiovascular profile, ${ }^{4,5}$ several cardiac abnormalities including left ventricular (LV) rotational abnormalities and dilated mitral annulus with impaired function could be demonstrated by three-dimensional (3D) speckle-tracking 
echocardiography (3DSTE). ${ }^{6,7}$ This relatively newly developed noninvasive imaging technique provides quantitative assessment of myocardial contractility by strain variables from acquired 3D echocardiographic datasets. ${ }^{8,9}$ The present study was designed to determine whether lipedema is associated with 3DSTE-derived LV deformation abnormalities, and to assess the effects of wearing MCS for 1 hour.

\section{2 | METHODS}

\section{1 | Patient population}

The present study comprised 19 female patients with stage 2 lipedema (mean age: $42.2 \pm 12.4$ years, body mass index: $34.1 \pm 7.0 \mathrm{~kg} / \mathrm{m}^{2}$ ), compared to 28 age-matched healthy female controls (mean age: 42.0 \pm 9.8 years, body mass index: $23.3 \pm 4.7 \mathrm{~kg} / \mathrm{m}^{2}$ ). None of the lipedema patients and controls had risk factors or any other disease that could theoretically affect the results. No medication was used by any subject. Lipedema was diagnosed and characterized on the basis of its typical clinical features (stove pipe legs, non-pitting edema, cuffing sign, spontaneous or minor trauma induced pain perception, easy bruising). ${ }^{3,10}$ Lipedema patients were referred by the Phlebolymphology Unit of the Department of Dermatology and Allergology, University of Szeged for routine echocardiographic examination extended with 3DSTE. Data originate from the MAGYAR-Path Study (Motion Analysis of the heart and Great vessels bY three-dimensionAl speckle-tRacking echocardiography in Pathological cases), which was organized to assess diagnostic and prognostic value of 3DSTE-derived variables. The current study was approved by the human research committee of the University of Szeged, Faculty of Medicine. It complied with the Declaration of Helsinki and all participants gave informed consent.

\section{2 | Two-dimensional Doppler echocardiography}

Complete two-dimensional (2D) transthoracic echocardiography examinations extended with Doppler measurements were performed in all healthy subjects and lipedema patients according to recent guidelines and practices. ${ }^{11}$ Examinations were performed with an Artida ultrasonographic system (Toshiba Medical Systems, Tokyo, Japan) with its PST-30SBP (1-5 MHz) phased-array transducer. Following chamber quantifications, transmitral E/A flow velocities were measured and valvular abnormalities were detected if present.

\section{3 | Three-dimensional speckle-tracking echocardiography}

The same echocardiography machine was used for 3DSTE assessments. Full volume 3D pyramid-shaped echocardiographic datasets were acquired by a PST-25SX matrix-array transducer. During data acquisition, six "as narrow as possible" wedge-shaped sub-volumes were recorded during six RR intervals and a single breath-hold. Then, the software merged them into a pyramid-shape 3D volume. Using these datasets, automatic offline analysis was performed in all cases. Apical four-chamber (AP4CH) and two-chamber (AP2CH) views and three cross-sectional views at different LV levels were automatically reconstructed from the 3D datasets using the 3D Wall Motion Tracking software version 2.7 (Toshiba Medical Systems, Tokyo, Japan) (Figure 1). Following optimization, markers were set to the edges of the mitral annulus and the apical LV endocardium by the examiners. Then, the software automatically reconstructed the LV endocardial surface and a 3D LV model was created. ${ }^{8,9}$

\section{4 | Assessment of 3DSTE-derived LV volumes and strains}

LV end-diastolic (EDV) and end-systolic (ESV) volumes, LV ejection fraction (EF), and LV mass were measured using the above-mentioned 3D LV model. Using the same acquired 3D echocardiographic datasets, several strains were measured as listed below ${ }^{8,9}$ :

Unidirectional (unidimensional) strains:

- radial strain (RS)-thickening and thinning of the segment

- longitudinal strain (LS)-lengthening and shortening of the segment

- circumferential strain (CS)-widening and narrowing of the segment

Multidirectional (multidimensional) or complex strains:

- area strain (AS) - combination of LS and CS

- 3D strain (3DS)-combination of RS, LS and CS

Segmental strain measurements were performed using the 16-segment LV model. Moreover, global and mean segmental strains were also calculated. ${ }^{8,9}$

\section{5 | Study protocol}

The study protocol was the same as reported in previous studies. ${ }^{6,7}$ First, 2D Doppler echocardiography was performed with 3DSTE in all patients with lipedema. Then, MCSs were put on and worn for 60 minutes. A second echocardiographic examination was performed before taking the MCSs off. During the 60-minute use of MCS, physical exercise, sitting with straight legs, standing and consuming any meal or beverage were not allowed. Stable room temperature $\left(21-22^{\circ} \mathrm{C}\right)$ and relative humidity (45-50\%) were ensured during the measurements. VenoTrain CuraFlow flat-knitted black-colored $\mathrm{ccl}$ 2 (23-32 mmHg) (Bauerfeind, Zeulenroda, Germany) stockings were applied consisting of $73 \%$ polyamide and $27 \%$ elastane. For interface pressure measurement between the skin and the compression material, Picopress device (Microlab Elettronica, Nicolò, Italy) was used at B1 point in standing position with a mean pressure of 22.12 $\pm 3.54 \mathrm{mmHg}$ in lipedema patients. ${ }^{12}$ 


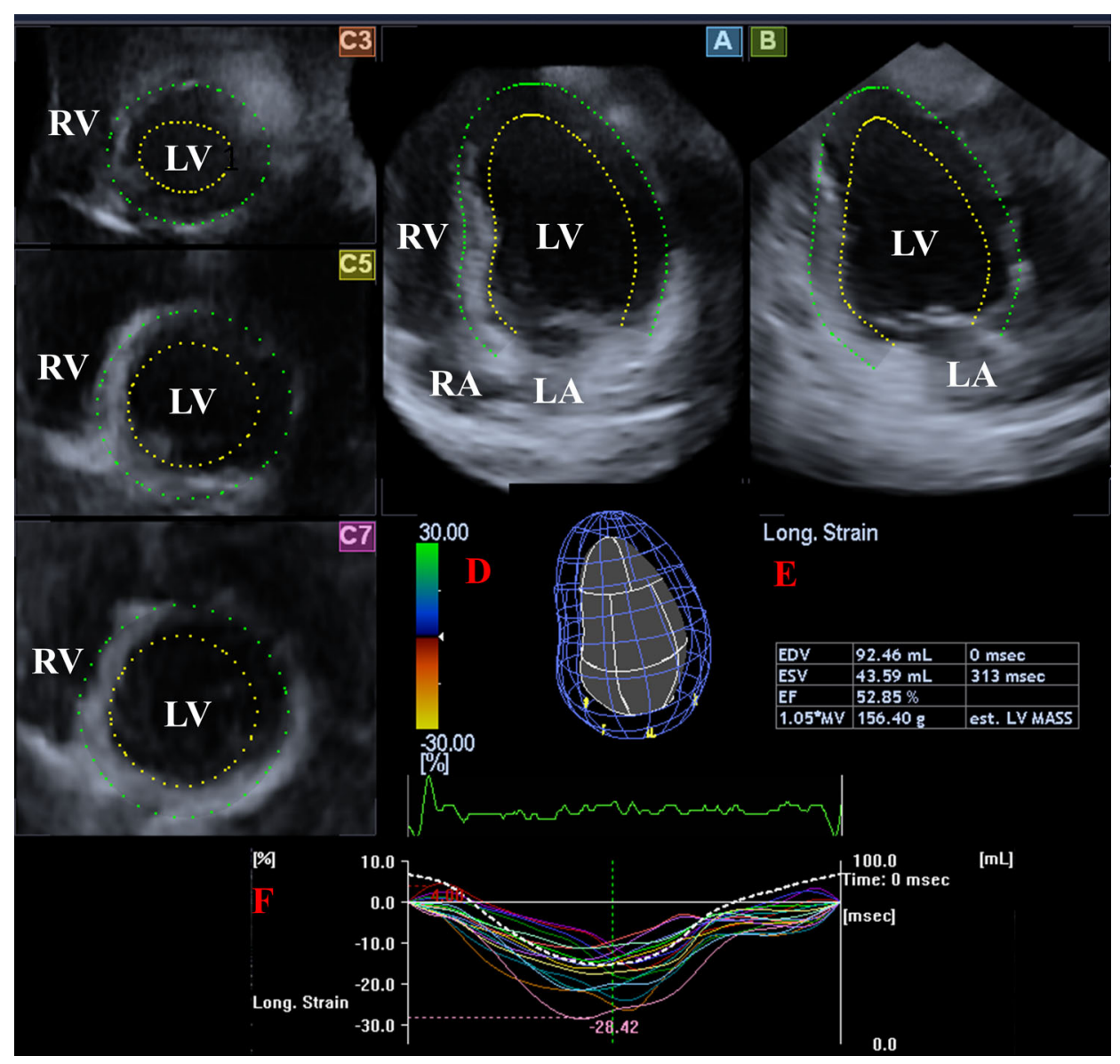

FIGURE 1 Apical four-chamber, $A$ and two-chamber, $B$ and crosssectional views at apical (C3), midventricular (C5) and basal (C7) left ventricular (LV) levels automatically selected from an acquired threedimensional echocardiographic dataset. LV three-dimensional cast, D; corresponding LV volumetric parameters, E; and segmental LV longitudinal strain curves, $\mathrm{F}$

\subsection{Statistical analysis}

Two-tailed tests were used for comparisons and $P$ value $<.05$ was defined as significant. Continuous data were shown as mean values \pm SD, while categorical data were presented as counts and percentages. To compare categorical variables, Fisher's exact test was used. For continuous variables, Shapiro-Wilk test was performed to test the variable distribution. If distribution was normal, Student $t$ test was used. If not, datasets were compared using Mann-Whitney-Wilcoxon test. RStudio (RStudio Team, RStudio Inc., Boston, Massachusetts, 2015) was used for statistical analysis.

Offline data extraction and analysis were performed by using the MatLab 8.6 (2015) software package (The MathWorks Inc., Natick, Massachusetts).

TAB LE 1 Two-dimensional echocardiographic data of patients with lipedema and controls

\begin{tabular}{|c|c|c|c|}
\hline & Controls & Lipedema patients & \\
\hline & $(n=28)$ & $(n=19)$ & $P$-value \\
\hline LA diameter (mm) & $34.4 \pm 4.1$ & $39.6 \pm 4.1$ & $<.001$ \\
\hline LV end-diastolic diameter (mm) & $45.8 \pm 3.2$ & $49.8 \pm 3.2$ & $<.001$ \\
\hline LV end-diastolic volume (ml) & $92.3 \pm 14.1$ & $119.2 \pm 18.1$ & $<.001$ \\
\hline LV end-systolic diameter (mm) & $32.7 \pm 11.1$ & $30.8 \pm 2.7$ & .9 \\
\hline LV end-systolic volume (ml) & $32.7 \pm 8.6$ & $38.3 \pm 8.8$ & .02 \\
\hline Interventricular septum (mm) & $8.8 \pm 1.3$ & $8.6 \pm 0.9$ & .4 \\
\hline LV posterior wall (mm) & $9.3 \pm 2.0$ & $8.7 \pm 0.9$ & .3 \\
\hline$E(\mathrm{~cm} / \mathrm{s})$ & $77.7 \pm 17.2$ & $86.0 \pm 18.0$ & .1 \\
\hline$A(\mathrm{~cm} / \mathrm{s})$ & $65.6 \pm 14.8$ & $72.9 \pm 18.9$ & .1 \\
\hline$E / A$ & $1.2 \pm 0.3$ & $1.3 \pm 0.4$ & .8 \\
\hline LV ejection fraction (\%) & $64.3 \pm 4.3$ & $68.3 \pm 3.6$ & .002 \\
\hline
\end{tabular}

Abbreviations: A, late transmitral flow velocity; E, early transmitral flow velocity; LA, left atrium; LV, left ventricle. 
TABLE 2 Comparison of three-dimensional speckle-tracking echocardiography-derived global and mean segmental left ventricular strains in patients with lipedema and controls

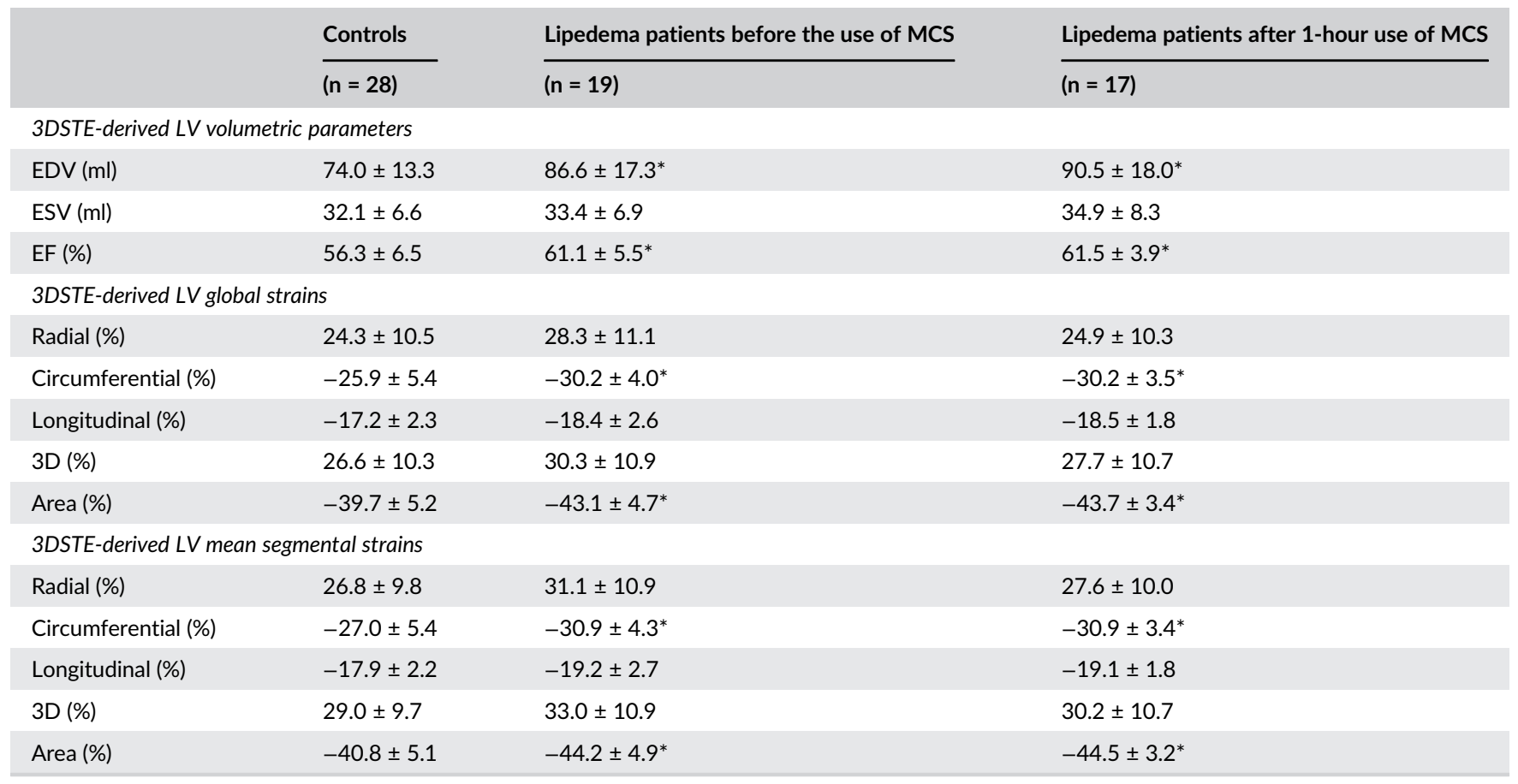

Abbreviations: EDV, end-diastolic volume; ESV, end-systolic volume; EF, ejection fraction; LV, left ventricular; MCS, medical compression stockings; 3DSTE, three-dimensional speckle-tracking echocardiography; 3D, three-dimensional.

${ }^{*} P<.05$ vs controls.

\section{3 | RESULTS}

\section{1 | Two-dimensional echocardiography}

Left atrial (LA) and LV dimensions, and LV ejection fraction were greater in lipedema patients than in controls, without significant difference in the other echocardiography variables (Table 1).

\section{2 | Three-dimensional speckle-tracking echocardiography}

Although 3DSTE-derived data acquisition was performed before and after the use of MCS, 3DSTE measurement could not be performed from the datasets acquired 1-hour after the use of MCS in 2 lipedema patients (Tables 2 and 3). Three-dimensional speckle-tracking echocardiography-derived volumetric measurements confirmed 2D echocardiographic assessments. Global and mean segmental LV-CS and LV-AS were greater in lipedema patients than in controls before the use of MCS. Following 1-hour use of MCS, none of global and mean segmental LV strains showed significant impairment or improvement in lipedema patients. At segmental analysis, only midventricular LV strains were significantly greater in lipedema patients than in controls. Apical segmental LV-CS, LV-AS and LV-3DS further increased after 1-hour use of MCS as compared to resting data in lipedema patients.

\section{DISCUSSION}

We used 3DSTE to assess global and segmental LV strains in lipedema patients and controls. This new non-invasive, angle-independent technique is feasible to quantify not only LV rotational abnormalities, but also global and segmental LV contractility by objective features called strains in the 3D space. ${ }^{8,9}$ It has been validated against other imaging techniques for LV strains and volumes, ${ }^{13-17}$ and normal reference values of LV strains are available. ${ }^{18}$

Previously, only cardiovascular profile data of lipedema patients was available in literature. ${ }^{4}$ Low prevalence of diabetes despite higher body mass index has been demonstrated, and majority of lipedema patients had a normal lipid profile. Only one-third of lipedema cases with stages 2 or 3 had hypertension according to literature. ${ }^{4}$ At this moment, only a limited number of clinical studies are available regarding lipedema-associated cardiovascular alterations. ${ }^{6,7,19}$ Although routine echocardiography shows no significant alterations, recent developments in imaging techniques generated new methodologies, including 3DSTE, allowing detailed analysis for deeper insights into the heart mechanics..$^{6-9}$ Not only LV rotational abnormalities and changes in contractility can be quantified with 3DSTE, but alterations of dimension and function of atria and valvular annuli can be characterized non-invasively in greater details. ${ }^{6-9}$

The result of the present study could be partially explained in the context of recent findings. Increased aortic stiffness has been demonstrated in lipedema. ${ }^{19}$ Moreover, LV apical rotation and twist were 
TAB LE 3 Comparison of three-dimensional speckle-tracking echocardiography-derived segmental left ventricular strains in patients with lipedema and controls

\begin{tabular}{|c|c|c|c|}
\hline & Controls & Lipedema patients before the use of MCS & Lipedema patients after 1 -hour use of MCS \\
\hline $\mathrm{RS}_{\text {basal }}(\%)$ & $33.1 \pm 15.5$ & $35.9 \pm 16.7$ & $30.9 \pm 14.2$ \\
\hline $\mathrm{RS}_{\text {apex }}(\%)$ & $16.6 \pm 7.7$ & $16.7 \pm 8.3$ & $20.4 \pm 9.0$ \\
\hline $\left.\mathrm{CS}_{\text {basal }} \%\right)$ & $-24.4 \pm 5.6$ & $-27.7 \pm 8.0$ & $-27.6 \pm 5.8$ \\
\hline $\mathrm{CS}_{\text {apex }}(\%)$ & $-31.6 \pm 9.8$ & $-33.2 \pm 8.0$ & $-34.9 \pm 6.2^{\dagger}$ \\
\hline $\mathrm{LS}_{\text {basal }}(\%)$ & $-22.1 \pm 4.6$ & $-19.9 \pm 5.0$ & $-21.0 \pm 3.8$ \\
\hline $\left.\mathrm{LS}_{\text {mid }} \%\right)$ & $-14.4 \pm 2.8$ & $-18.0 \pm 3.3^{*}$ & $-17.3 \pm 4.5$ \\
\hline $\mathrm{LS}_{\text {apex }}(\%)$ & $-16.9 \pm 5.3$ & $-20.0 \pm 4.9$ & $-19.1 \pm 2.2$ \\
\hline $\mathrm{AS}_{\text {basal }}(\%)$ & $-40.4 \pm 6.6$ & $-41.3 \pm 8.9$ & $-42.2 \pm 6.5$ \\
\hline $\mathrm{AS}_{\text {mid }}(\%)$ & $-38.0 \pm 6.4$ & $-44.8 \pm 4.6^{*}$ & $-43.8 \pm 5.4^{*}$ \\
\hline $\mathrm{AS}_{\text {apex }}(\%)$ & $-44.4 \pm 10.7$ & $-47.6 \pm 9.4$ & $-48.9 \pm 5.5^{\dagger}$ \\
\hline
\end{tabular}

Abbreviations: AS, area strain; CS, circumferential strain; LS, longitudinal strain; MCS, medical compression stockings; RS, radial strain; 3DS, three-dimensional strain.

${ }^{*} P<.05$ vs controls.

${ }^{\dagger} P<.05$ vs lipedema POST group.

found to be significantly reduced in lipedema patients with severe LV rotational abnormalities in some (14\%) cases. ${ }^{6}$ Due to a known close relationship between LV rotational mechanics and aortic stiffness, even in healthy subjects, ${ }^{20}$ these abnormalities could be partially explained by each other. Moreover, direct effects of noncardiomyocytes with mesenchymal cells and adipose tissue depositions could not be excluded, which can be the topic of further investigations. ${ }^{3}$ In spite of these findings, LV-EF was increased in lipedema patients as compared to matched controls in the present study, which could be explained by increased LV strains suggesting hypercontractility of the LV in certain directions to maintain pumping function as a compensatory effect despite LV rotational abnormalities. LV strains are diminished in most of diseases, but in some disorders or states (for instance in acromegaly or in elite athletes), ${ }^{21,22}$ similarly to the findings of the present study, hypercontractility (increased global or regional strains) has been demonstrated and considered as a compensating mechanism. ${ }^{21}$ These alterations suggest specific lipedemarelated/associated cardiomyopathy, which warrants further investigation in this common but underdiagnosed disorder.

\section{1 | Limitations}

There are several limiting factors affecting the results:

- Lipedema is a rare disease that can be either underdiagnosed or misdiagnosed with similar diseases like obesity and lymphedema.
Therefore, recruiting lipedema patients without known comorbidity is difficult, resulting in the relatively small population sample of lipedema patients. ${ }^{1-3}$

- The duration of the disease and its effects on the evaluated variables were not examined.

- There were some important technical limitations, including lower spatial and temporal resolution of 3DSTE as compared to 2D echocardiography. ${ }^{8}$

- LV strains show some vendor-dependency, and differences are present in 2DSTE- and 3DSTE-derived variables as well. ${ }^{17,23} \mathrm{How}$ ever, 3DSTE-derived LV volumetric and strain assessments have been validated, which is why the present study included no reproducibility study. ${ }^{13-17}$ Moreover, normal reference values of 3DSTEderived LV strains are available. ${ }^{18}$

- We did not perform 3DSTE-derived assessment of other heart chambers. $^{8}$

- Finally, comparison of 2D echocardiography-derived LV volumes and that of measured by 3DSTE was not the aim of this study.

\section{5 | CONCLUSION}

Lipedema patients showed greater LV strains than controls, which could be a compensatory mechanism maintaining LV pumping function. Wearing MCS for 1 hour had no global effects on LV strains. 


\section{CONFLICT OF INTEREST}

The authors declare that they have no conflict of interest.

\section{ETHICAL APPROVAL}

All procedures performed in studies involving human participants were in accordance with the ethical standards of the institutional and/or national research committee and with the 1964 Helsinki declaration and its later amendments or comparable ethical standards.

\section{INFORMED CONSENT}

Informed consent was obtained from all individual participants included in the study.

\section{ORCID}

Attila Nemes (D) https://orcid.org/0000-0002-7570-6214

\section{REFERENCES}

1. Szél E, Kemény L, Groma G, Szolnoky G. Pathophysiological dilemmas of lipedema. Med Hypotheses. 2014;83:599-606.

2. Bauer AT, von Lukowicz D, Lossagk $K$, et al. New insights on lipedema: the enigmatic disease of the peripheral fat. Plast Reconstr Surg. 2019;144:1475-1484.

3. Forner-Cordero I, Szolnoky G, Forner-Cordero A, Kemény L. Lipedema: an overview of its clinical manifestations, diagnosis and treatment of the disproportional fatty deposition syndrome - systematic review. Clin Obes. 2012;2:86-95.

4. Buso G, Depairon M, Tomson D, et al. Lipedema: a call to action! Obesity (Silver Spring). 2019;27:1567-1576.

5. Torre YS, Wadeea R, Rosas V, Herbst KL. Lipedema: friend and foe. Horm Mol Biol Clin Investig. 2018;33. https://doi.org/10.1515/hmbci2017-0076.

6. Nemes A, Kormanyos A, Domsik P, et al. Left ventricular rotational mechanics differ between lipedema and lymphedema: insights from the three-dimensional speckle tracking echocardiographic MAGYARpath study. Lymphology. 2018;51:102-108.

7. Nemes A, Kovács Z, Kormányos Á, et al. The mitral annulus in lipedema: insights from the three-dimensional speckle-tracking echocardiographic MAGYAR-Path Study. Echocardiography. 2019;36: 1482-1491.

8. Nemes A, Kalapos A, Domsik P, Forster T. Three-dimensional speckletracking echocardiography - a further step in non-invasive threedimensional cardiac imaging. Orv Hetil. 2012;153:1570-1577.

9. Nemes A, Forster T. Recent echocardiographic examination of the left ventricle - from M-mode to 3D speckle-tracking imaging. Orv Hetil. 2015;156:1723-1740.

10. Lee BB, Andrade M, Antignani PL, et al. International Union of Phlebology. Diagnosis and treatment of primary lymphedema. Consensus document of the International Union of Phlebology (IUP)-2013. Int Angiol 2013;32:541-574.

11. Lang RM, Badano LP, Mor-Avi V, et al. Recommendations for cardiac chamber quantification by echocardiography in adults: an update from the American Society of Echocardiography and the European Association of Cardiovascular Imaging. Eur Heart J Cardiovasc Imaging. 2015;16:233-270.
12. Partsch H, Mosti G. Comparison of three portable instruments to measure compression pressure. Int Angiol. 2010;29:426-430.

13. Seo $\mathrm{Y}$, Ishizu T, Enomoto $\mathrm{Y}$, et al. Validation of 3-dimensional speckle tracking imaging to quantify regional myocardial deformation. Circ Cardiovasc Imaging. 2009;2:451-459.

14. Nesser HJ, Mor-Avi V, Gorissen W, et al. Quantification of left ventricular volumes using three-dimensional echocardiographic speckle tracking: comparison with MRI. Eur Heart J. 2009;30:15651573.

15. Kleijn SA, Brouwer WP, Aly MF, et al. Comparison between threedimensional speckle-tracking echocardiography and cardiac magnetic resonance imaging for quantification of left ventricular volumes and function. Eur Heart J Cardiovasc Imaging. 2012;13: 834-839.

16. Kleijn SA, Aly MF, Terwee CB, van Rossum AC, Kamp O. Reliability of left ventricular volumes and function measurements using threedimensional speckle tracking echocardiography. Eur Heart J Cardiovasc Imaging. 2012;13:159-168.

17. Saito $\mathrm{K}$, Okura $\mathrm{H}$, Watanabe $\mathrm{N}$, et al. Comprehensive evaluation of left ventricular strain using speckle tracking echocardiography in normal adults: comparison of three-dimensional and two-dimensional approaches. J Am Soc Echocardiogr. 2009;22:1025-1030.

18. Kleijn SA, Pandian NG, Thomas JD, et al. Normal reference values of left ventricular strain using three-dimensional speckle tracking echocardiography: results from a multicentre study. Eur Heart J Cardiovasc Imaging. 2015;16:410-416.

19. Szolnoky G, Nemes A, Gavallér H, Forster T, Kemény L. Lipedema is associated with increased aortic stiffness. Lymphology. 2012;45: 71-79.

20. Nemes A, Kalapos A, Domsik P, Lengyel C, Orosz A, Forster T. Correlations between echocardiographic aortic elastic properties and left ventricular rotation and twist--insights from the three-dimensional speckle-tracking echocardiographic MAGYAR-Healthy Study. Clin Physiol Funct Imaging. 2013;33:381-385.

21. Kormányos Á, Domsik P, Kalapos A, et al. Active acromegaly is associated with enhanced left ventricular contractility: Results from the three-dimensional speckle-tracking echocardiographic MAGYAR-Path Study. Rev Port Cardiol. (in press).

22. Nemes A, Kalapos A, Domsik P, et al. Is elite sport activity associated with specific supranormal left ventricular contractility? (Insights from the three-dimensional speckle-tracking echocardiographic MAGYARSport Study). Int J Cardiol. 2016;220:77-79.

23. Gayat E, Ahmad H, Weinert L, et al. Reproducibility and inter-vendor variability of left ventricular deformation measurements by threedimensional speckle-tracking echocardiography. J Am Soc Echocardiogr. 2011;24:878-885.

How to cite this article: Nemes A, Kormányos Á, Domsik P, et al. Are increased left ventricular strains compensatory effects in lipedema? Detailed analysis from the threedimensional speckle-tracking echocardiographic MAGYAR-Path Study. J Clin Ultrasound. 2020;48:470-475. https://doi.org/10.1002/jcu.22855 